\title{
Correction to: Ground truth to fake geographies: machine vision and learning in visual practices
}

\author{
Abelardo Gil-Fournier ${ }^{1}\left(\mathbb{D} \cdot\right.$ Jussi Parikka ${ }^{1,2} \mathbb{D}$ \\ (C) Springer-Verlag London Ltd., part of Springer Nature 2020
}

\section{Correction to: Al \& SOCIETY} https://doi.org/10.1007/s00146-020-01062-3

In the original publication of the article, the following paragraphs have been indented wrongly in the published article.

The first paragraph of the article which should be indented.

"Knowing how to discern a groundless image from an image that is nothing but a blow is an entire art in itself"-Jean-Luc Nancy (2005: 25)."

Paragraphs which should not be indented:

"This doubling is both philosophical and technical, as our article aims to show. This argument relates essentially to a range of contemporary data and computational techniques and shifts as part of the broader framework of how we engage with questions of AI-such as different machine learning techniques-as part of operational images: images that do not primarily represent but operate in scientific, military, and other technical systems and institutions (see Farocki 2004).

This article is structured around three key points: first, to address the scope of the notion of ground truth, we map the shift from the truth of the ground to pattern recognition as a significant transformation that also relates to questions of machine vision and machine learning techniques (even if we are not able to go into technical specifics in this article). Second, we show how, from the recognition of patterns, we can move to the building of datasets as a relevant part of the infrastructures of ground truth and machine vision. Third, we look at examples of synthetic geographies as experiments that help to understand the ensemble of images in which the ground becomes synthetized with meaningful aesthetic and epistemological consequences.

At stake in our discussion is the claim that ground truth is read from a mass of images, instead of comparatively off the ground. This leads to the question of how these concepts and terms relate to the contemporary situation of machine vision and, more specifically, machine learning and the production of synthetic landscapes, as we argue at the end of the article."

Publisher's Note Springer Nature remains neutral with regard to jurisdictional claims in published maps and institutional affiliations.

Abelardo Gil-Fournier

abelardo.gilfourniermartinez@famu.cz

Jussi Parikka

j.parikka@soton.ac.uk

FAMU, Prague, Czechia

2 University of Southampton, Southampton, UK 